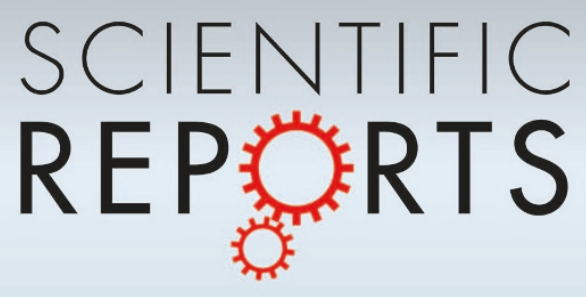

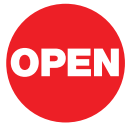

SUBJECT AREAS:

MAGNETIC MATERIALS

AND DEVICES

ENGINEERING

MATERIALS PHYSICS

ELECTRONIC MATERIALS AND

DEVICES

Received

18 January 2012

Accepted

9 July 2012

Published

25 July 2012

Correspondence and requests for materials should be addressed to

J.Y. (jiandong.ye@ anu.edu.au) or K.L.T. (eleteokl@nus.edu.sg).

\section{Spin-polarized Wide Electron Slabs in Functionally Graded Polar Oxide} Heterostructures

\author{
Jiandong Ye ${ }^{1,2}$, Sze Ter Lim ${ }^{3}$, Michel Bosman ${ }^{4}$, Shulin Gu', Youdou Zheng' ${ }^{1}$, Hark Hoe Tan², \\ Chennupati Jagadish ${ }^{2}$, Xiaowei Sun ${ }^{5} \&$ Kie Leong Teo ${ }^{3}$
}

${ }^{1}$ School of Electronics Science and Engineering, Nanjing University, 210093 (PR China), ${ }^{2}$ Department of Electronic Materials Engineering, Research School of Physics and Engineering, The Australia National University, Canberra, 0200, (Australia), ${ }^{3}$ Department of Electrical and Computer Engineering, National University of Singapore, 117576 (Singapore), ${ }^{4}$ Institute of Materials Research and Engineering, A-STAR (Agency for Science, Technology, and Research), 3 Research Link, 117602 (Singapore), ${ }^{5}$ School of Electrical \& Electronic Engineering, Nanyang Technological University, Nanyang Avenue, 639798 (Singapore).

We report on the high mobility wide electron slabs with enhanced correlation effects by tailoring the polarization effects in a functionally graded $\mathrm{ZnMgO} / \mathrm{ZnO}$ heterostructures. The characteristics of three-dimensional (3D) spreading electrons are evidenced by the capacitance-voltage profiling and the quantization of 3D Fermi surface in magneto-transport measurements. Despite the weak spin-orbit interaction, such electron slabs are spin-polarized with a large zero-field spin splitting energy, which is induced by the carrier-mediated ferromagnetism. Our results suggest that the vast majority of electrons are localized at the surface magnetic moment which does not allow spin manipulations, and only in the region visited by the itinerant carriers that the ferromagnetic exchange interactions via coupling to the surface local moments contribute to the spin transport. The host ferromagnetism is likely due to the formation of $\mathrm{Zn}$ cation vacancies on the surface regime induced by the stabilization mechanism and strain-relaxation in $\mathrm{ZnMgO}$ polar ionic surface.

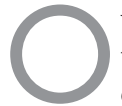

wing to the richness of their intrinsic properties, transition metal oxides can bring novel functionalities to modern electronics. Recent technical advances in the fabrication of atomically abrupt oxide interface enable the formation of high mobility two-dimensional electron gases (2DEG) and trigger intense research of unusual electronic properties in deliberately designed oxide structures ${ }^{1-5}$. In particular, the emerging phenomena of metal-insulator transition and quantum Hall ferromagnet have been observed in a diluted $\mathrm{ZnMgO} / \mathrm{ZnO}$ strongly correlated $2 \mathrm{DEG}^{1,6}$. Of interest to high frequency device application, it is essential to dilute the carrier density in device channel because that the build-up of non-equilibrium polar optical phonons will limit the high-field velocity of carriers in high density 2DEG. It is also expected that the reduction of charge carrier density will further increase the correlation effects, resulting in enhancements of the electron mass and spin susceptibility ${ }^{1}$. Spreading carriers into a wide compositionally graded heterostructure is an effective mean to dilute the carrier density, as demonstrated in the graded $\mathrm{AlGaN} / \mathrm{GaN}$ and graded $\mathrm{AlGaAs} / \mathrm{GaAs}$ wide parabolic quantum wells ${ }^{7-9}$. Such a low density three-dimensional (3D) electron slab is also a ubiquitous paradigm in solidstate physics and quantum chemistry ${ }^{10}$. If the spin degree of freedom is incorporated, spin physics as a new frontier will offer more intriguing studies in spintronics. It is also promising to develop novel spintronic devices by utilizing the desirable spin-polarization feature of high mobility electron system ${ }^{11}$. Recently, the compositionally graded $\mathrm{ZnMgO}$ structures have been utilized to reduce the internal electric field of quantum wells and enhance the surface Schottky barrier ${ }^{12-13}$. Here, we explore the polarization effects in a functionally graded pseudomorphic $\mathrm{ZnMgO} / \mathrm{ZnO}$ heterostructure. By tailoring the bandgap and polarization effects, a diluted three-dimensional electron slab (3DES) can be achieved without compromising the high mobility characteristics. The intriguing phenomena of spin-polarization and carrier-mediated ferromagnetism in such a strongly correlated electron system have been investigated. The formation mechanism and quantum transport of 3DES are examined in terms of band structure and polarization effect. 


\section{Results}

Tailoring the polarization effects in functionally graded polar oxides. A compositional grading feature in $\mathrm{ZnMgO}$ layer will result in a non-vanishing divergence of polarization. It is well understood from the theory of dipole engineering established by D. Jena et $\mathrm{al}^{7-8,14}$. Figure 1 (a) shows the polarization field in the [0001] direction for a linearly graded $\mathrm{ZnMgO} / \mathrm{ZnO}$ heterostructure studied in this work. The distribution of polarization dipoles and the resultant carriers are depicted schematically in Figure 1 (b) and (c), respectively. For a compressively strained $\mathrm{ZnMgO}$ layer, both spontaneous polarization and piezoelectric polarization should be taken into account while in a completely relaxed thick $\mathrm{ZnO}$ underlayer, the spontaneous polarization is only considered. In Fig. 1 (b), each unit cell is assumed to contain a charge dipole due to spatial separation of centroids of the electron clouds and the nuclei. The dipoles sheets in each unit cell neutralize each other in bulk $\mathrm{ZnO}$. However, the strength of the dipole changes with increasing $\mathrm{Mg}$ composition, breaking the local charge neutrality in the $\mathrm{ZnMgO}$ layer. To visualize the microscopic landscape of polarization effect, we divide the $\mathrm{Zn}_{1-\mathrm{x}} \mathrm{Mg}_{\mathrm{x}} \mathrm{O}$ layer into $\mathrm{N}$ unit cells with a $\mathrm{Mg}$ composition linearly increasing from the interface $\left(z=z_{0}\right)$ to the top surface $(z=0)$. The unit number $N$ is equal to $d / c$, where $d$ is the thickness of graded layer, and $c$ is the length of a unit cell. For the $i^{\text {th }}$ unit cell, $\mathrm{Mg}$ composition is $\frac{N-i}{N-1} z_{0}$, and the unbalanced bound sheet charge is equal to $\left|P_{S P+P E}^{i} / q\right|$ and $-\left|P_{S P+P E}^{i} / q\right|$ at the upper and lower surface, respectively. Due to the gradient polarization discontinuity $\Delta P\left(=\left|\Delta P_{S P}\right|-\left|P_{P E}\right|\right)<0^{4}$, the unbalanced net bound charges are positive with its sheet density of $\left(P_{S P+P E}^{i}-P_{S P+P E}^{i+1}\right) / q$ at the interface between the $i$ th and $(i+1)^{\text {th }}$ unit cells. As a result, the bound positive polarization charges are spread throughout the linearly graded layer with a volume density of $N_{D \pi}=\nabla P=\sigma_{\pi} / d q^{7}$. Consequently, these fixed positive charges will attract mobile electrons to satisfy the Poisson's equation and local charge neutrality in the bulk. Thus, gradient polarization magnitude introduces a three-dimensional mobile electron slab (3DES).

Composition gradient in $\mathrm{ZnMgO} / \mathrm{ZnO}$ heterostructure. In this work, the polarization gradient is experimentally realized by utilizing the compositional pulling effect in $\mathrm{ZnMgO} / \mathrm{ZnO}$ heterostructure grown on sapphire under thermal equilibrium conditions by metal-organic vapor phase epitaxy (MOVPE) technique ${ }^{15}$. The details are given in the Method section. The single heterostructure (sample A) consists of a $\mathrm{Zn}_{1-{ }_{x}} \mathrm{Mg}_{\mathrm{x}} \mathrm{O}(75 \mathrm{~nm})$ on $\mathrm{ZnO}$ template with $x=0.18$ at the topmost surface, while the double heterostructure (sample B) has a sandwich feature: graded $-\mathrm{Zn}_{0.8} \mathrm{Mg}_{0.2} \mathrm{O}(60 \mathrm{~nm}) /$ $\mathrm{ZnO}(30 \mathrm{~nm}) /$ graded $-\mathrm{Zn}_{0.85} \mathrm{Mg}_{0.15} \mathrm{O}(90 \mathrm{~nm})$. Figure 2 (a) shows a cross-sectional image of sample $\mathrm{B}$ obtained by high-angle annular dark-field cross-sectional scanning transmission electron microscopy. The sandwiched $\mathrm{ZnO}$ layer appears as bright because the heavier $\mathrm{Zn}^{2+}$ ions appear brighter than the $\mathrm{Mg}^{2+}$ ions. The atomic lattice in both $\mathrm{ZnMgO}$ and $\mathrm{ZnO}$ layers is clearly seen (right enlarged image in Fig. 2 (a)), indicative of state-of-the-art epitaxial heterostructure formed. Fig. 2 (b) shows energy-dispersive X-ray (EDX) chemical maps of $\mathrm{Mg}$ and $\mathrm{Zn}$, and as well as monochromated lowloss electron energy-loss spectroscopy (EELS) data. The contrast in the maps illustrates the composition distribution of $\mathrm{Zn}$ and $\mathrm{Mg}$ in the heterostructure and the spatially resolved EELS spectra exhibit a stepwise absorption feature resulting from the electronic bandgap onset. The EELS spectra are displayed at $5 \mathrm{~nm}$ intervals, with $\mathrm{ZnO}$ region as marked by red lines. The extracted bandgap profile at the right of Fig. 2 (b) was taken from all $1 \mathrm{~nm}$-spaced EELS spectra. The profile shows a linear bandgap-energy reduction, which is consistent with the $\mathrm{Zn}$ and $\mathrm{Mg}$ distributions of $\mathrm{ZnMgO}$ layers. This indicates that the gradient distribution of $\mathrm{Mg}$ in $\mathrm{ZnMgO}$ layers has been achieved. This compositional gradient phenomenon in $\mathrm{ZnMgO}$ layer is known as compositional pulling or lattice latching effect, which is related to the misfit strain in the films ${ }^{16-17}$. From the viewpoint of thermodynamics, the biaxial compressive strain will pull the $\mathrm{Mg}$ adatoms from the growing front surface of $\mathrm{ZnMgO}$ in the initial stage to reduce the deformation energy. As growth continues, a graded strain reduction allows the subsequent deposition of a $\mathrm{ZnMgO}$ layer with high $x$. Due to the gradual distribution of strain throughout the whole $\mathrm{ZnMgO}$ layer and not accumulated at the sharp interface, the $\mathrm{ZnMgO}$ layer can grow coherently in the critical range without generating new misfit dislocations. The slope of grading can be tuned by various thermodynamic growth conditions such as temperature and $\mathrm{Mg} / \mathrm{Zn}$ gas-phase mole ratio ${ }^{18}$.

Strain evolution and polarity stabilization in graded $\mathrm{ZnMgO}$ layer. The piezoelectric polarization field is sensitive to the strain evolution in graded $\mathrm{ZnMgO}$ layer, which can be examined by means of high resolution x-ray diffraction. Figure 3 (a) and (b) a

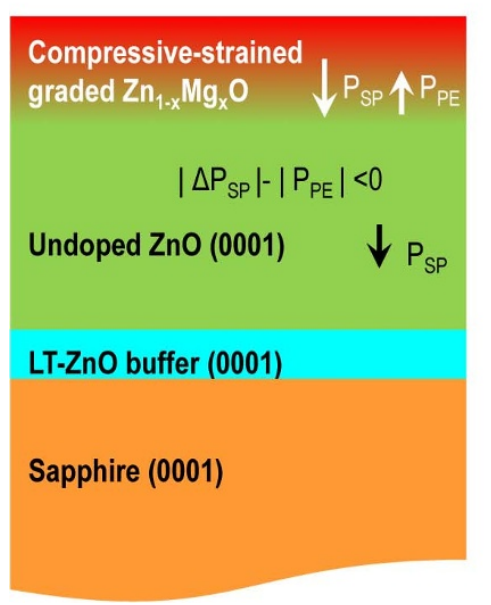

b

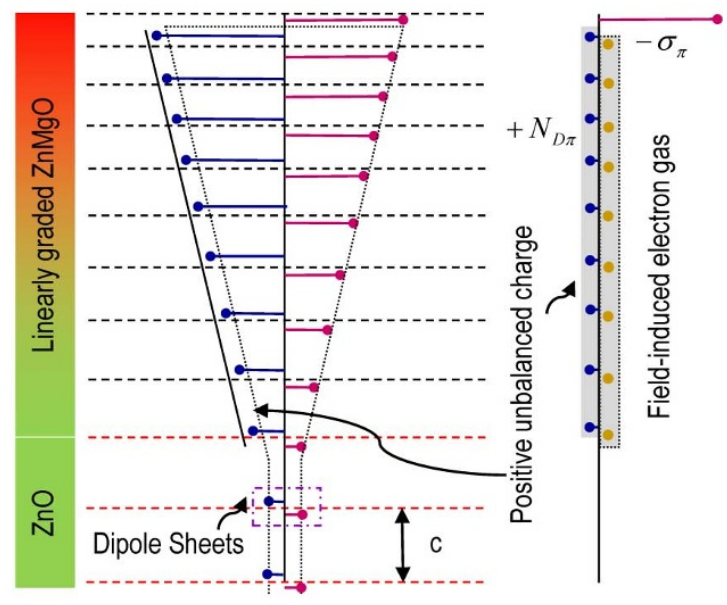

Figure $1 \mid$ (a) Polarization field directions, (b) schematic distribution of polarization dipole and (c) distribution of resultant carriers in the linearly graded $\mathrm{ZnMgO} / \mathrm{ZnO}$ heterostructure in the [0001] orientation. $P_{S P}$ and $P_{P B}$ represent the spontaneous and piezoelectric polarizations, respectively. The parameter $\mathrm{c}$ is the lattice constant, $\sigma_{\pi}$ is the polarization-induced charges on top of the surface and $N_{D \pi}$ is the sheet density of positive unbalanced polarization charges. 

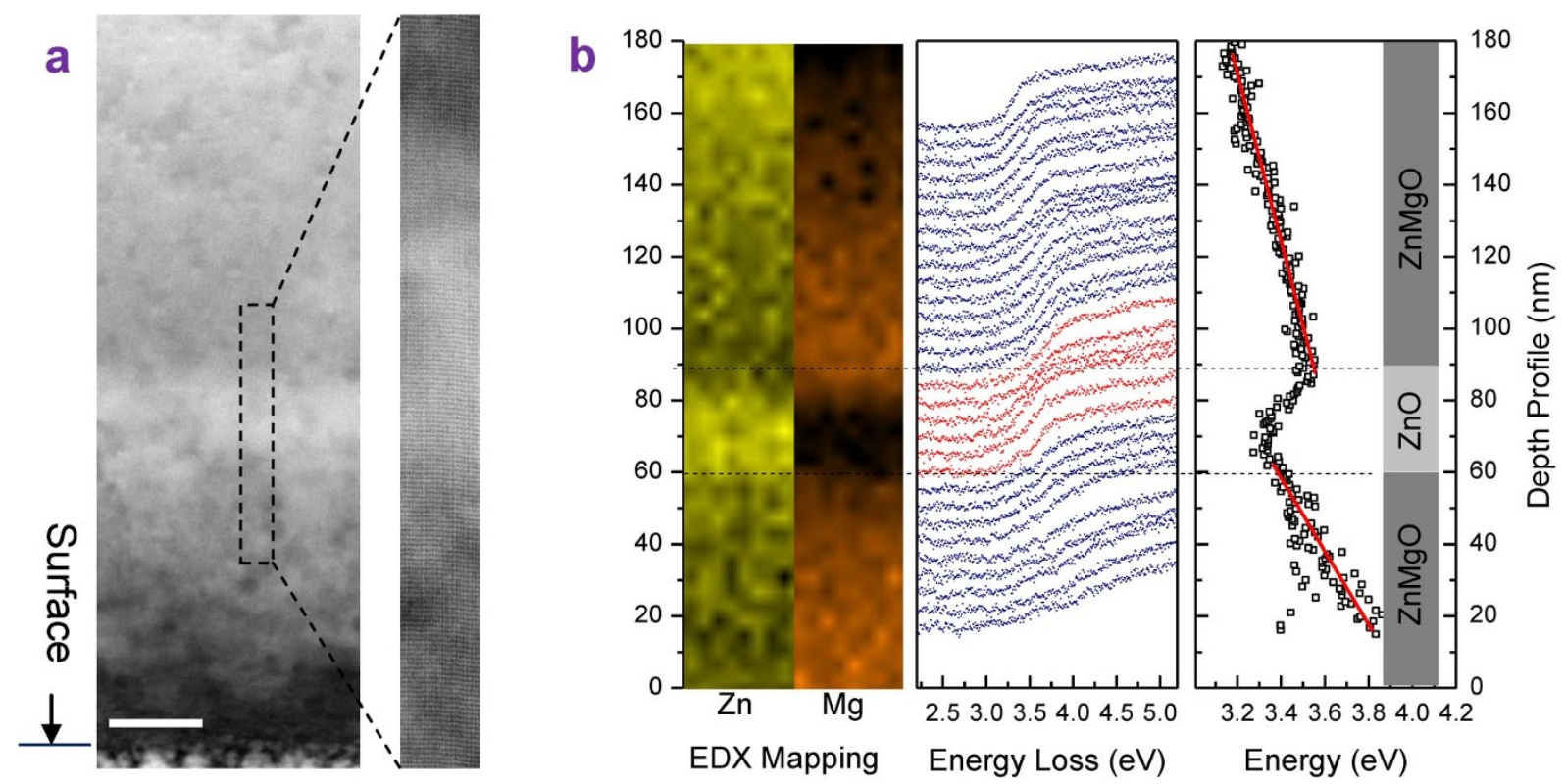

Figure $2 \mid$ (a) Cross-sectional STEM HAADF image and the corresponding higher magnification image of the boxed region; (b) (left to right) EDX chemical maps with projected $\mathrm{Mg}$ and $\mathrm{Zn}$, monochromated low-loss EELS spectra and the extracted bandgap energy profile of $\mathrm{ZnMgO} / \mathrm{ZnO} / \mathrm{ZnMgO}$ double heterostructures (sample B).

illustrate the reciprocal-space maps (RSMs) recorded in the vicinity of (105) asymmetric reflection of $\mathrm{ZnMgO} / \mathrm{ZnO}$ heterostructures of sample A and B, respectively. In both samples, identical reciprocal coordinate $q_{x}$ are obtained for both $\mathrm{ZnMgO}$ and $\mathrm{ZnO}$ template which indicate that $\mathrm{ZnMgO}$ barrier layers are coherently strained to the underlying $\mathrm{ZnO}$ layers. The lattice constant of the pseudomorphic $\mathrm{ZnMgO}$ barrier along the c-axis decreases less gradually than the relaxed films, suggesting that the alloying barriers suffer from the in-plane compressive (out-of-plane tensile) $\operatorname{strain}^{19}$. The elongated and broadened reciprocal lattice point of $\mathrm{ZnMgO}$ along $q_{z}$ vector indicates a gradual decrease of the lattice constant along c-axis, clearly exhibiting the presence of $\mathrm{Mg}$ composition gradient.

We employed X-ray photoemission spectroscopy (XPS) to probe the chemical and electronic nature of $\mathrm{ZnMgO}$ surface. The shape of valence band (VB) XPS in Figure 4 (a) clearly exhibits Zn-polarity, in which $\mathrm{O} 2 p$ valence band states are distinguished ${ }^{20}$. It is known that $\mathrm{ZnMgO}$ epilayers exhibit higher spontaneous polarization effects than $\mathrm{ZnO}$. Due to large electronegativity of oxygen, the polar surfaces

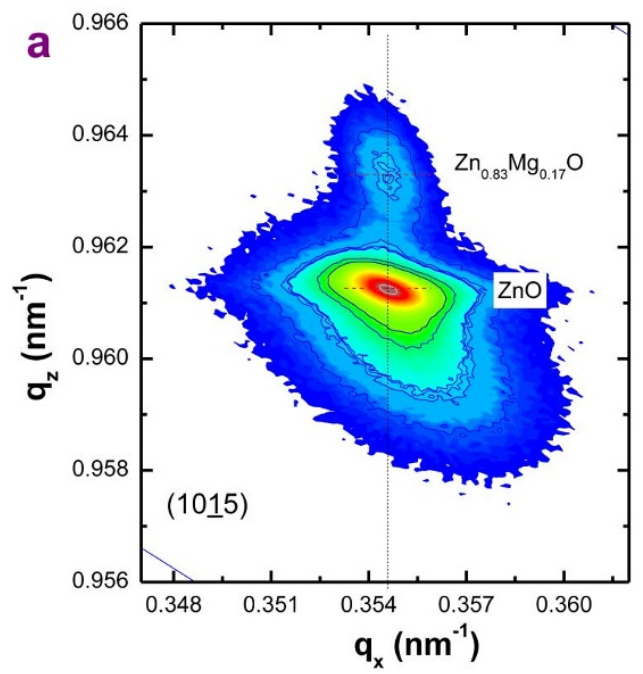

are electrostatically unstable, and the surface reconstruction or hydroxide absorption is considered crucial to stabilize the polar surface ${ }^{21-23}$. In checking for $\mathrm{O} 1 \mathrm{~s}$ core-level spectrum, only one dominant peak at $531.00 \mathrm{eV}$ was observed in Fig. 4(b) due to oxygen in the bulk, and thereby the absorbed $\mathrm{OH}$ groups were not obviously present in such clean surface. Thus, the surface reconstruction with formation of structural defects could be the dominant stabilization mechanism. The analysis of XPS indicates that the atomic ratio of $[\mathrm{O}] /[\mathrm{Zn}+\mathrm{Mg}]$ on $\mathrm{Zn}$-polar $\mathrm{ZnMgO}$ epilayer is $49.70 / 48.84$, slightly deviated from the stoichiometry. The modification of the surface stoichiometry for the stability of (0001)-Zn surface is also related to the redistribution of the surface charges through the formation of O-terminated defects. The atomic force microscopic image in Figure 4(c) exhibited the triangular jagged step edges and tiny pits were dominant on the surface graded $\mathrm{ZnMgO}$ layer and the mean surface roughness is only $0.42 \mathrm{~nm}$ over $3 \mu \mathrm{m} \times 3 \mu \mathrm{m}$ area. These triangular shaped features are similar to those observed by Dulub et $\mathrm{a}^{21}$, which are not present on the O-polar or nonpolar faces. In fact,

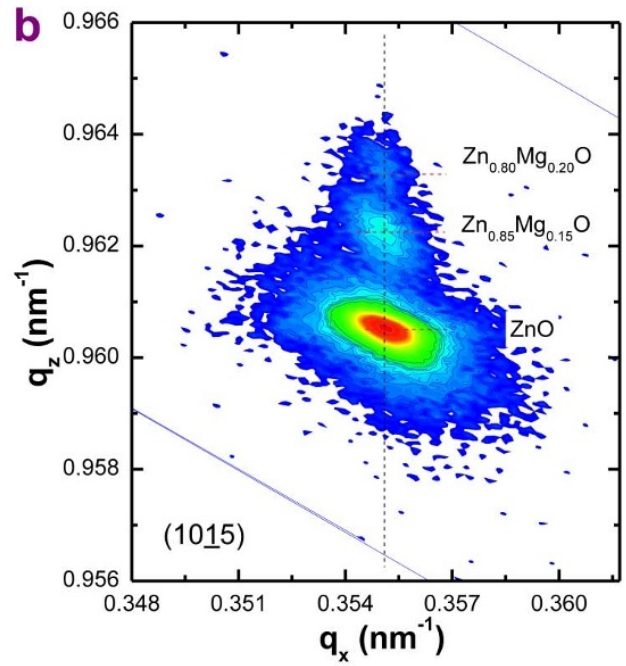

Figure 3 | (105) asymmetric RSMs of sample A (a) and sample B (b). 

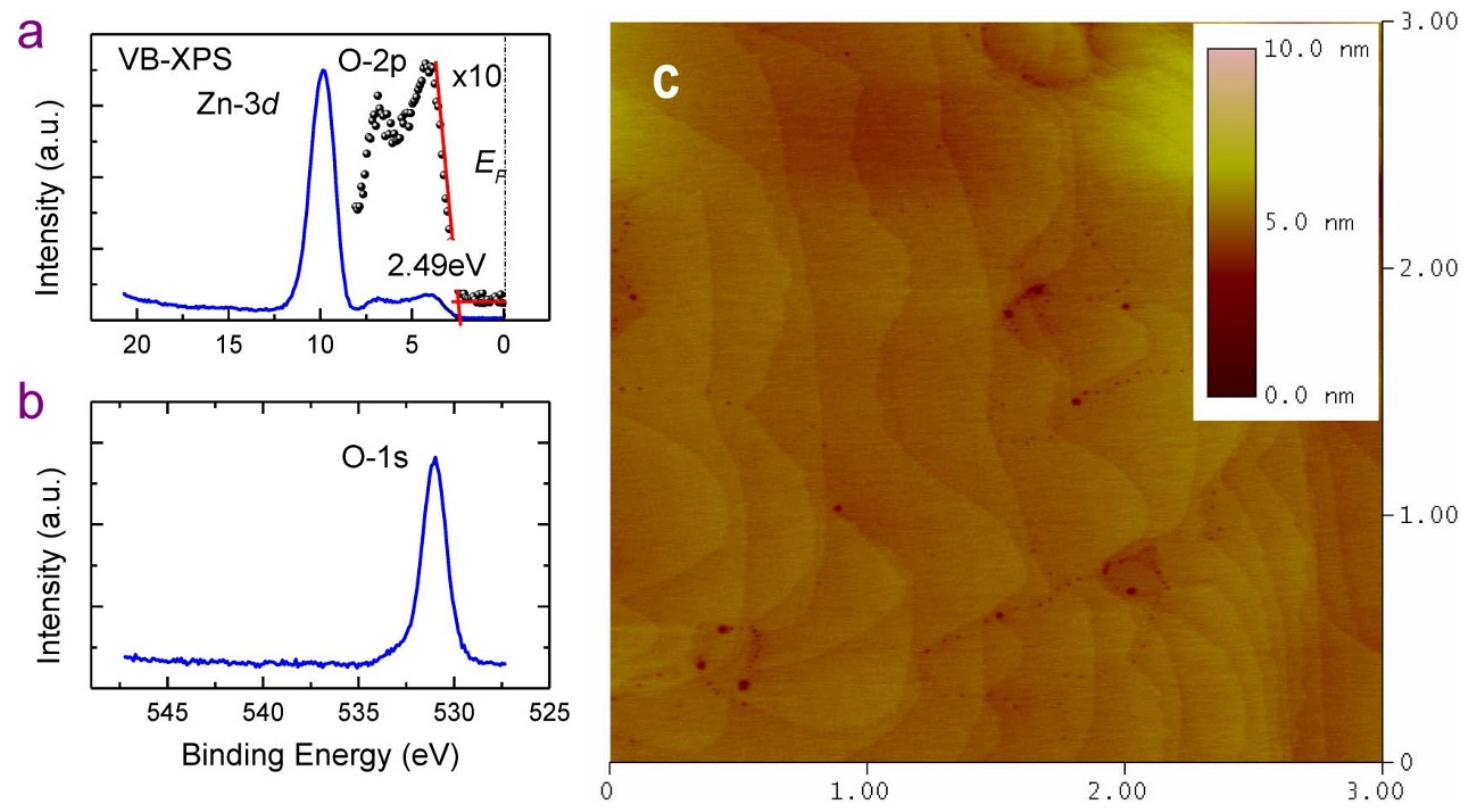

Figure $4 \mid$ (a) The valance-band and (b) O 1s core-level x-ray photoelectron spectrum of sample A calibrated by carbon 1s (284.6 eV); (c) AFM images of (a) $\mathrm{ZnMgO} / \mathrm{ZnO}$ double heterostructure (sample $\mathrm{B}$ ).

during the step-flow growth under thermal equilibrium, the different growth speed is determined by different dangling bond densities at two kinds of step edges, leading to the jagged structures. The theoretical calculations and experimental results suggests that these step edges are O-terminated and thereby result in the overall decrease of the surface $\mathrm{Zn}$ concentration, which in turn build in a net-electric field to compensate the electrostatic surface instability ${ }^{21-22}$. On the other hand, the lattice-mismatch induced compressive strain in $\mathrm{ZnMgO}$ will tend to relax and give priority to the formation of tiny pits at the step edges of terraces. These pits are also considered to be clusters of negative charged $\mathrm{Zn}$ vacancies with O-terminated hexagonal cavities ${ }^{23}$. Therefore, the formation of structural defects $\mathrm{Zn}$ vacancies or even vacancy clusters are energetically favored and abundant on the surface regime.

Polarization charge distribution and carrier profile of 3DES. The polarization tailoring effect is verified by performing self-consistent Poisson and Schrodinger band calculations based on the surface charge model $^{24}$. Figure 5 (a) and (b) show the calculated band diagrams and charge profiles at zero bias for both structures, and the real carrier profiles of samples derived from capacitance-voltage $(\mathrm{C}-\mathrm{V})$ measurements. The surface potential is determined to be $1.2 \mathrm{eV}$ by VB-XPS in Fig. 4 (a), where the Fermi level is located at energy level of $2.49 \mathrm{eV}$ above the maximum of valence-band and the corresponding bandgap of $\mathrm{ZnMgO}$ on the surface is $3.69 \mathrm{eV}$. The polarization constants used in the calculation are obtained from Ref. ${ }^{25}$. Taking both the spontaneous and piezoelectric polarization effects into account, the polarization discontinuity in a coherently strained $\mathrm{ZnMgO}$ is expressed as $P^{S P+P E}(x)=0.029 x(\mathrm{C} / \mathrm{m})^{26}$. For a linear gradient from $\mathrm{ZnO}$ to $\mathrm{ZnMgO}$ over length $d$ with $x(z)=x_{0} z$ l $d$ ( $x_{0}$ is the $\mathrm{Mg}$ composition on the topmost surface), the volume density of itinerant carriers is given as $n_{3 D}=N_{D \pi}=0.029 x_{0} / d$. It is found that the calculated carrier profile coincide well with those derived from $\mathrm{C}-\mathrm{V}$ profiling. As expected, mobile carriers are accumulated within the graded barrier layer with a depletion layer $d_{\text {depl }}$ near the surface due to upward band bending. The resultant carrier sheet density is approximated to be $n_{3 D} \times\left(d-d_{\text {depl }}\right)$ with sheet density of $1.48 \times 10^{12}$ and $3.89 \times 10^{12} \mathrm{~cm}^{-2}$ for samples $\mathrm{A}$ and $\mathrm{B}$, respectively. It is noted that the spreading widths of electron slabs are larger than the thermal de-Broglie wavelength $\left(\lambda_{d B}=h / \sqrt{2 m * k_{B} T} \approx 21 \mathrm{~nm}\right.$ at $\left.300 \mathrm{~K}\right)$ or Fermi wavelength $\left(\lambda=2 \pi / k_{F} \approx 26 \mathrm{~nm}\right)$ for the given carrier density of $n_{3 D}=4.77 \times 10^{17} \mathrm{~cm}^{-3}$ in sample A. The electronic system here is therefore expected to have a $3 \mathrm{D}$ single particle energy spectrum, and the carriers act just like bulk doped electrons but with high mobility.

Quantum transport of spin-polarized high mobility carriers. Figure 6 shows the temperature dependent Hall effect data for both samples $\mathrm{A}$ and $\mathrm{B}$ and a $\mathrm{ZnO}$ template for reference. At high temperature, the differences in sheet carrier concentration and mobility are not well distinguished between heterostructures and $\mathrm{ZnO}$ template because the parallel conduction path served by the $\mathrm{ZnO}$ underlayer dominate the Hall signal. As a result, a huge deviation of $n_{\mathrm{s}}$ obtained by the overall Hall data $\left(1.16 \times 10^{14} \mathrm{~cm}^{-2}\right)$ and $\mathrm{C}-\mathrm{V}$ profiling $\left(1.48 \times 10^{12} \mathrm{~cm}^{-2}\right)$ is observed. Once at low temperature, the parallel conduction path is greatly suppressed because most of electrons are frozen onto their parent donors in $\mathrm{ZnO}$ underlayer, and the resultant concentration decreases greatly and approaches to the real density of electron gas housed in the graded ZnMgO layer, as seen in Fig. 5 (b). We note that the parallel conduction path from $\mathrm{ZnO}$ underlayer is not completely suppressed, and thus, the carrier concentration of 3DES determined by the Hall Effect is still overestimated while the mobility is underestimated. Alternatively, we have performed the cyclotron resonance measurements by using terahertz time-domain magnetospectroscopy at $1.4 \mathrm{~K}$. Since the terahertz conductivity is a local probe of carrier dynamics ${ }^{27}$, this may provide us more accurate information on the real 3DES. Our results show that for sample A, the carrier concentration, effective mass and mobility of the electrons are obtained to be: $n_{s}=1.8 \times 10^{12} \mathrm{~cm}^{-2} ; m^{*}=0.33 m_{e}$; $\mu=3500 \mathrm{~cm}^{2} / V s$. We note that the carrier mobility for the polarization-doped $3 \mathrm{DES}$ is more than two orders of magnitude improvement as compared to undoped $\mathrm{ZnO}$ template. The low mobility of only $12 \mathrm{~cm}^{2} / \mathrm{Vs}$ for undoped $\mathrm{ZnO}$ is caused by ionized impurity scattering. While in 3DES case, the carriers spreading in a wide graded $\mathrm{ZnMgO}$ layer are ionized by the non-vanishing polarization field. Owing to the removal of ionized impurity scattering, no sign of electron freeze-out at low temperature is observed and thus gives rise to metallic state of 3DES with high mobility. The main limitation mechanism in 3DES is alloy disorder 

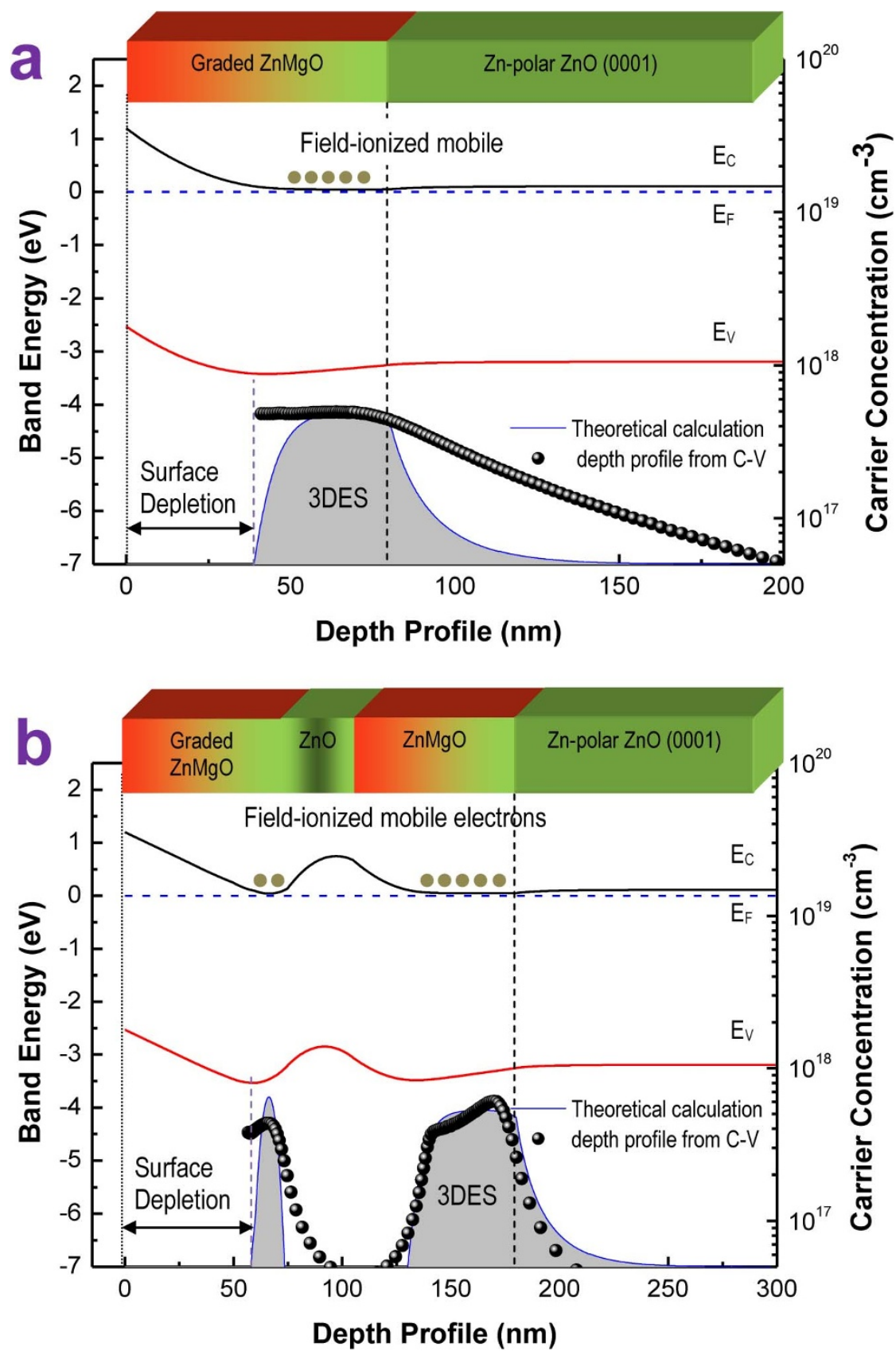

Figure 5 The calculated band diagrams and charge profiles at zero bias and carrier depth profile derived from C-V measurements for (a) sample A and (b) sample B.

scattering, since the entire electron system completely resides in the graded $\mathrm{ZnMgO}$ layer ${ }^{14}$. As the volume density of the 3DES is diluted, the electron system can no longer fully screen the random disordered alloy potential, leading to the increased possibility of alloying disorder scattering. Furthermore, random spatial electric dipole moment due to the replacement of $\mathrm{Zn}$ by $\mathrm{Mg}$ atoms will result in a non-periodic electronic potential ${ }^{28}$, thereby enhancing the dipole scattering of mobile electrons.

Next, we turn to the quantum transport properties of the 3DES. If ignoring the quantum electron-electron correlation effects, the 3DES investigated here has 3D Landau-level spectrum without variations of the density of states due to dimensional quantization across the spreading layer, as confirmed by CV profiling. However, below a certain temperature, the characteristic coherent length $\left(l_{T}=\sqrt{D \hbar / k_{B} T}\right.$ $\approx 160 \mathrm{~nm}$ ) for coherent transport is larger than the electron spreading width, and thus, the system becomes $2 \mathrm{D}$ for coherent phenomena in the diffusive transport ${ }^{29}\left(D=v_{F} l\right.$ is the diffusion coefficient along the magnetic field, $l=v_{F} \tau=55.7 \mathrm{~nm}$ is the mean free path for sample A). Figure 7 shows the angular-dependent magnetoresistance $\left(\mathrm{R}_{\mathrm{xx}}\right)$ and Hall resistance $\left(R_{x y}\right)$ as a function of magnetic field for sample $A$. When the magnetic field is perpendicular to the sample surface $(z / /$ $B, \theta=0^{\circ}$ where $z$ is the growth direction), the structure of $\mathrm{R}_{\mathrm{xx}}$ and $\mathrm{R}_{\mathrm{xy}}$ resemble the Shubnikov-de Haas $(\mathrm{SdH})$ oscillation and quantum Hall effect (QHE) for a 2D electron system. Above $\sim 3 \mathrm{~T}$, well-resolved $\mathrm{SdH}$ oscillations and quantum Hall plateaus are observed, and the minima of $\rho_{x x}$ coincide well with the quantized Hall resistivity $\left(\rho_{x y}\right)$ plateaus of $h /\left(v q^{2}\right)$, where $v$ is the Landau filling index. However, compared to the conventional QHE in 2DEG, neither the steps of $\mathrm{R}_{\mathrm{xy}}$ plateaus are completely flat nor the minima of $\mathrm{R}_{\mathrm{xx}}$ are very deep. This is due to the energy gap that is not yet fully developed in a finite magnetic field $\mathrm{d}^{27}$. The negative parabolic magnetoresistance characteristic is observed at low field. It is related to the weak localization and 

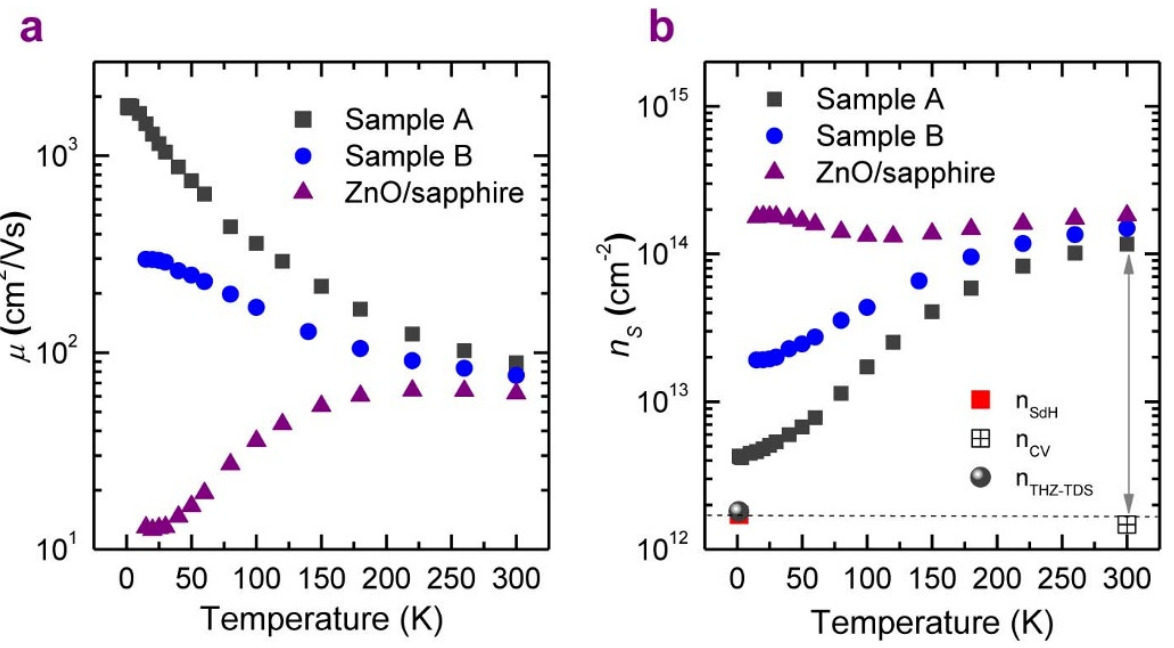

Figure $6 \mid$ (a) Mobility and (b) sheet carrier concentration of $\mathrm{ZnMgO} / \mathrm{ZnO}$ heterostructures (samples $\mathrm{A}$ and $\mathrm{B}$ ) as well as the reference $\mathrm{ZnO}$ template.

electron-electron interaction effects, which only occur in the coherent diffusive transport regime ${ }^{30}$. By rotating the magnetic field away from the perpendicular orientation, the minima in $\mathrm{R}_{\mathrm{xx}}$ are strongly suppressed. With the magnetic field parallel to the sample surface $\left(z \perp B, \theta=90^{\circ}\right)$, the $\mathrm{SdH}$ oscillation can still be observed to start with an onset at higher field, which unambiguously implies the quantization of three-dimensional bulk Fermi surface. However, the in-plane oscillation amplitude decreased greatly, which could be ascribed to the finite ratio of the electron spreading width with respect to the magnetic length $\left(l_{0}=\sqrt{\hbar / e B}\right)$. The similar features of $\mathrm{SdH}$ oscillations under in-plane magnetic field have also been observed in AlGaAs/ $\mathrm{GaAs}$ wide parabolic quantum well and heavily doped GaAs layer ${ }^{29,31}$.

The theoretical expression of the envelope function of the $\mathrm{SdH}$ oscillation in $3 \mathrm{D}$ case can be given as ${ }^{31}$

$\frac{R_{x x}-R_{0}}{R_{0}}=\frac{2}{5}\left(\frac{\hbar \omega_{c}}{2 E_{F(3 D)}}\right)^{1 / 2} \frac{A_{T}}{\sinh A_{T}} \exp \left(-\frac{\pi}{\omega_{c} \tau}\right) \cos \left(\frac{E_{F(3 D)}}{\hbar \omega_{c}}-\frac{\pi}{4}\right)$

Where $A_{T}=\left(2 \pi^{2} k_{B} T\right) /\left(\hbar \omega_{c}\right), E_{F(3 D)}=\frac{\hbar^{2}}{2 m^{*}}\left(3 \pi^{2} n_{3 d}\right)^{\frac{2}{3}}$ is the Fermi energies of 3D states, $\omega_{c}=(e B) /(m c)$ is the cyclotron frequency, $m$ is the electron effective mass, $\tau$ is the quantum scattering time, and $R_{0}$ represents the classical resistance in zero magnetic fields. When the high mobility system is at extreme low temperature of $1.4 \mathrm{~K}$, the magnetoresistance exhibited a weakly modulated $\left(\sim B^{1 / 2}\right)$ dependence of $\cos \left(\frac{E_{F(3 D)}}{\hbar \omega_{c}}-\frac{\pi}{4}\right)$, and the period of the oscillatory cosine term yields the carrier density. We employed the standard Fast Fourier Transform (FFT) method to extract the periodicity of $\mathrm{SdH}$ oscillatory component and the FFT power spectrum is shown in Fig. 7 (b). Two peaks are clearly resolved with their locations at $f_{1}$ $=51.92 \mathrm{~T}$ and $f_{2}=19.26 \mathrm{~T}$, together with a shoulder peak on the left side of $f_{1}$ peak. The peak fitting result indicates that the shoulder peak is superimposed by the second harmonic peak $2 f_{2}$ and the dominant peak $f_{1}$. If we assume that the two FFT peaks arise from the population of two subbands, the sheet carrier concentration is then given as $n_{s}=2 e\left(f_{1}+f_{2}\right) / \hbar=3.44 \times 10^{12} \mathrm{~cm}^{-2}$, while if considering the spin-polarized Fermi surface, the value is $n_{s}=e\left(f_{1}+f_{2}\right) / \hbar=$ $1.72 \times 10^{12} \mathrm{~cm}^{-2}$. The latter value agrees well with the concentrations
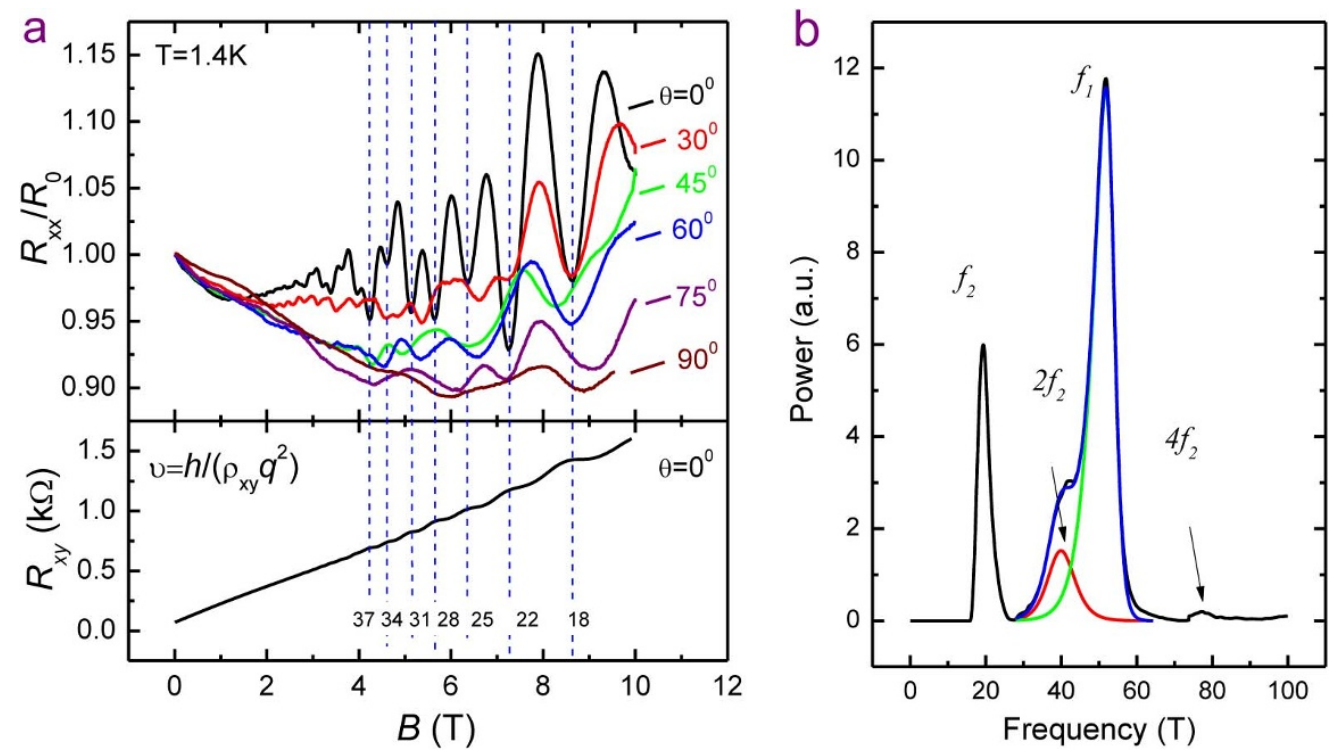

Figure 7 | (a) Angular-dependent normalized magnetoresistance $R_{x x} / R_{0}$ versus $B$ and Hall resistivity $R_{x y}$ of sample A as a function of magnetic field ( $z / /$ $B, \theta=0^{\circ}$ ) at $1.4 \mathrm{~K}$; (b) FFT power spectrum of $\mathrm{R}_{\mathrm{xx}}$ versus $\mathrm{B}$ at $1.4 \mathrm{~K}$. 
derived by means of capacitance-voltage $\left(1.48 \times 10^{12} \mathrm{~cm}^{-2}\right.$ at RT) as well as cyclotron resonance $\left(1.8 \times 10^{12} \mathrm{~cm}^{-2}\right.$ at $\left.1.4 \mathrm{~K}\right)$. Thus, the resultant volume carrier concentration $\left(n_{3 d}\right)$ is calculated be $4.77 \times 10^{17} \mathrm{~cm}^{-3}$. Correspondingly, we can conclude that such diluted 3DES are partially spin polarized with spin polarization degree $\zeta\left(\equiv\left|n_{\uparrow}-n_{\downarrow}\right| / n\right)$ of 0.46 , and a large zero-field spin-splitting energy $\Delta E\left(=2 \pi \hbar^{2}\left(n_{\uparrow}-n_{\downarrow}\right) / m^{*}\right)$ of $11.5 \mathrm{meV}$. Here $n_{\uparrow}$ and $n_{\downarrow}$ are the spin-up and spin-down carrier densities and $m^{*}=0.33 m_{e}$.

\section{Discussion}

In the framework of Landau's Fermi liquid approach, the carriercarrier interaction effects can be described using a dimensionless Wigner-Seitz parameter $r_{s}$, which is defined as the ratio of Coulomb interaction to Fermi energy. The $r_{s}$ value has a strong dependence on carrier concentration with the relationship given as $\frac{4}{3} \pi a_{B}^{3} r_{s}^{3}=n_{3 d}$ in $3 \mathrm{D}$ case $^{32}$. Here, $a_{B}=\left(\varepsilon / m_{b}\right) a_{b}=1.54 \mathrm{~nm}$, which gives the effective Bohr radius with dielectric constant of $\varepsilon=8.45$ and effective mass of conduction-band electron $\left(m_{b}=0.29 m_{e}\right)$. Given the volume carrier concentration of $n_{3 d}=4.77 \times 10^{17} \mathrm{~cm}^{-2}$, $r_{s}$ in sample $\mathrm{A}$ is 6.25 . We note that it is rather difficult to achieve high $r_{s}$ value while still maintaining a high mobility in other semiconductor materials. Due to very small lattice mismatch, the pseudomorphic growth of $\mathrm{Zn}_{0.8} \mathrm{Mg}_{0.2} \mathrm{O}$ on $\mathrm{ZnO}$ can be achieved with critical thickness up to $700 \mathrm{~nm}^{19}$. Thus, it is possible to further tune down the gradient of $\mathrm{Mg}$ composition in a wider thickness range and the polarization-induced charge density of high mobility $3 \mathrm{DES}$ can be diluted lower than $10^{16} \mathrm{~cm}^{-3}$. In this situation, the corresponding value of $r_{s}>22$ can be reached, and new physics such as metalinsulator transition or even quantum Hall ferromagnetic states can possibly be observed.

Knowing the interaction effects of carriers in diluted 3DES, we can now re-examine the feature of huge spin splitting energy of $11.5 \mathrm{meV}$ in such intrinsic $\mathrm{ZnMgO} / \mathrm{ZnO}$ system. The well-known Rashba spinorbital coupling (SOC) effect due to inversion asymmetry are usually responsible for the zero-spin splitting phenomenon observed in $\mathrm{AlGaAs} / \mathrm{GaAs}$ material systems. However, the SOC coefficient is very small in $\mathrm{ZnO}$ (about $1.1 \mathrm{meV} \AA$ ) ${ }^{33}$, and thus, it cannot explain our result. In fact, as the magnetic field is applied parallel to the surface of sample A, the Lorenz force and weak localization due to orbital effects are eliminated, and the distinct negative magnetoresistance is observed. This strongly suggests the inherent magnetic order of $3 \mathrm{DES}^{34}$. The scenario of magnetic impurity induced spinpolarization can be ruled out because no magnetic impurities are detected by SIMS measurement within its detection sensitivity. Thus, the spin polarization observed in the magneto-transport is due to the ferromagnetic phase of electrons in 3DES, similar to the observations in 2DEG confined in non-magnetic polar oxides $\left(\mathrm{LaAlO}_{3} / \mathrm{SrTiO}_{3}\right)$ heterostructures ${ }^{35}$. Theoretical study has predicted an itinerant 3DES will undergo a transition to a ferromagnetic phase in the limit of a large ratio of Columbic to kinetic energy $\left(r_{s}>50\right)^{36}$. By comparison, $r_{s}$ for $3 \mathrm{DES}$ investigated here is not large enough to induce the quantum Hall ferromagnetic phase, and therefore, we infer that the spin polarization is originated not only from the intrinsic electron-electron interaction but also includes other effects.

Figure 8(a) shows the magnetic hysteresis loops of sample A and $\mathrm{ZnO}$ template measured at $5 \mathrm{~K}$ after diamagnetic and paramagnetic subtraction. The sample A exhibits an enhanced ferromagnetism (FM) as compared to the $\mathrm{ZnO}$ template and the remanent moment at zero field is $2 \times 10^{-6} \mathrm{emu} / \mathrm{cm}^{2}$ after subtracting the contribution from $\mathrm{ZnO}$ template. We determine the net spin polarized electrons by adding the net moments of all dipoles in 3DES, yielding $n_{S} \cdot \zeta=6.8 \times 10^{11} \mu_{B} \mathrm{~cm}^{-2}$ ( $\mu_{B}$ is the Bohr magneton). The net magnetic moment induced by delocalized electrons in 3DES is estimated to be three orders of magnitude lower than the zero-field remanent moment of $1.8 \times 10^{14} \mu_{B} \mathrm{~cm}^{-2}$ measured by SQUID. Such a difference is also observed in $\mathrm{LaAlO}_{3} / \mathrm{SrTiO}_{3}$ system ${ }^{37}$. It implied that the host ferromagnetism does not allow spin manipulation and the vast majority of localized spins have no contribution to the transport properties.

So far, the issue of intrinsic ferromagnetism in nonmagnetic oxides nanostructures and thin films is still controversial. Nonetheless, most of the recent reports indicate that the main source of the magnetic moment is likely due to the unpaired $2 p$ electrons at oxygen site surrounding the $\mathrm{Zn}$ vacancies, and vacancy-vacancy interaction in the ground state will enhance the magnetic moments ${ }^{38}$. Our XPS and AFM results imply that $\mathrm{Zn}$ vacancies are expected to be majority of structrual defects at surface regime driven by the polarity stablization and strain relaxation mechanisms. The theoretical calculation predicted that the surface ferromagnetism in polar $\mathrm{ZnO}$ surface is in turn a plausible general stablization mechansim of polar ionic surfaces ${ }^{39}$. Since $\mathrm{Zn}$ vacancies amount are less in the bulk and do not lead to magnetism because of their stronger orbital hybridizations, we believe that $\mathrm{Zn}$ vacancies at the surface regime is most likely the main
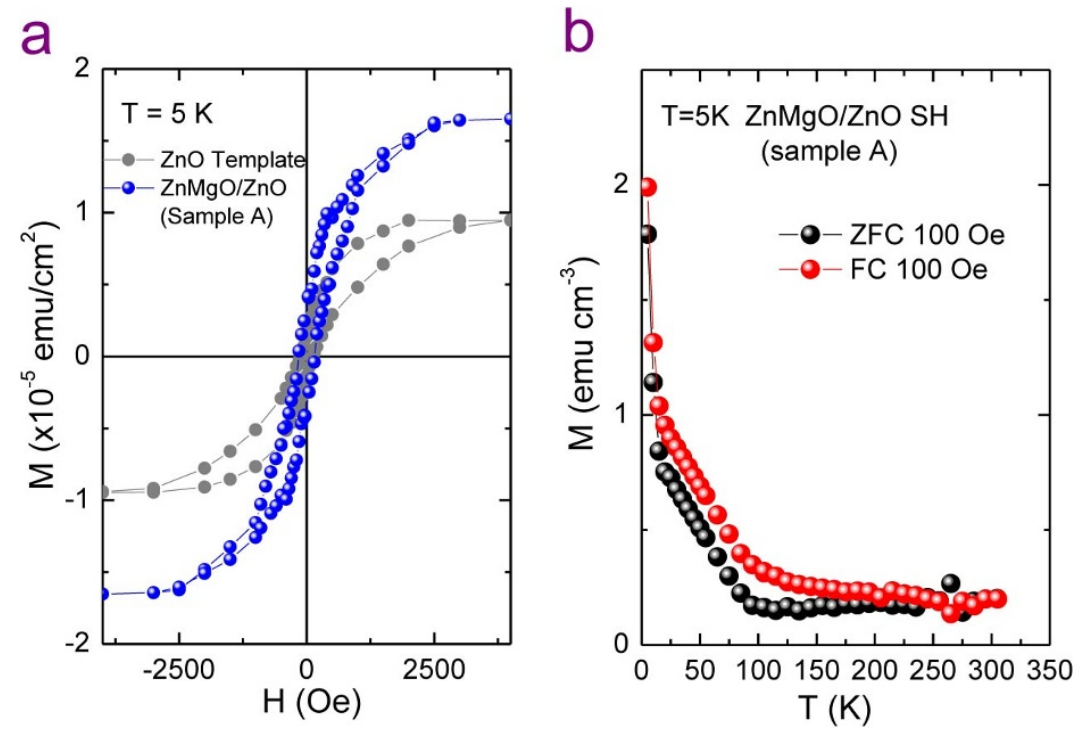

Figure $8 \mid$ (a) The M-H loop after diamagnetic and paramagnetic subtraction for sample A and referenced $\mathrm{ZnO}$ template measured at $5 \mathrm{~K}$; (b) zerofield cooled (ZFC) and field-cooled (FC) magnetization curves of sample A. 
source of ferromagnetism. This also agrees with various observations of enhanced ferromagnetism in nanostructured and ultra-thin films in which materials are non-magnetic but exhibit high surface-volume ratio ${ }^{38}$. It was also well supported by the fact that the ferromagnetism is enhanced with the increasing of $\mathrm{ZnMgO}$ thickness and $\mathrm{Mg}$ composition in a series of controlled samples that we have investigated (see Supporting Information).

Figure 8 (b) shows the zero-field cooled (ZFC) and field cooled (FC) magnetization as a function of temperature for sample A. The measurement was carried out while warming the sample from 5 to $300 \mathrm{~K}$ using a small applied magnetic field (100 Oe). The magnetization decreases quickly till $90 \mathrm{~K}$ and remains weak up to $300 \mathrm{~K}$. The magnitude and temperature dependence of the magnetic response in ZFC and FC curves indicated that the vast majority of the carriers are localized at the surface local moments. Although the valence electrons in $\mathrm{O} 2 p$ orbitals are less delocalized than those in $d$ or $f$ orbitals in transition metals ${ }^{38}$, large thermal energy is necessary to activate the electrons localized in such deep level $\mathrm{Zn}$ vacancies. Thus, at extreme low temperature, these localized spins are immobile and have no contribution to the transport properties. However, driven by the non-vanishing polarization field across the graded $\mathrm{ZnMgO}$ layer, a small fraction of carriers at the surface regime above the Fermi level are migrated and transferred into the conduction band to form 3DES, similar to the transfer process of 2DEG at the $\mathrm{LaAlO}_{3} / \mathrm{SrTiO}_{3}$ and $\mathrm{ZnMgO} / \mathrm{ZnO}$ interface ${ }^{26,40}$. In this region visited by the itinerant carriers the ferromagnetic exchange interactions are strong via coupling to the surface local moments. The large coherent length and mean free path of electrons also ensure low possibility of spin-flip during carrier migration, thus giving rise to the spin-polarization feature. Note that although $r_{s}$ in 3 DES is not high enough for ferromagnetic phase transition, the diluted electron density definitely favors the spin polarization phenomenon. This is also consistent with small spin polarization degree of 0.35 for the $2 \mathrm{DEG}$ confined in $\mathrm{ZnMgO} / \mathrm{ZnO}$ sharp interface with same $\mathrm{Mg}$ composition of $x=$ $0.18^{15}$. Additionally, as the magnetization originates mainly from the surface regime, the interplay of surface charges and spins with the absorbed charged species is expected to be strong, and thus, the $\mathrm{ZnO}$ based open gate transistor can be utilized for biocompatible medicinal applications.

In summary, we have demonstrated the tailoring of polarization effects in functionally graded polar oxides to produce high mobility 3DES without impurity doping. The ability to tune carrier density in graded $\mathrm{ZnMgO} / \mathrm{ZnO}$ heterostructure provides the opportunity to explore strong correlation effects in the area of quantum transport and spintronics. The important feature of spin polarization is the result of exchanging interaction of itinerant electrons and vacancies induced local magnetic moments. The enhanced correlation effects in such diluted electron system will facilitate the spin polarization, and thus, it could benefit novel all-oxide electronic and spintronic devices for high-field operation.

\section{Methods}

Sample growth. $\mathrm{ZnMgO} / \mathrm{ZnO}$ heterostructures were grown on sapphire (0001) substrates using the Aixtron close-coupled showerhead (CCS) MOVPE system ${ }^{13}$. Dimethylzinc (DMZn) and bis(methlycyclopentadienyl) magnesium $\left(\mathrm{MeCp}_{2} \mathrm{Mg}\right)$ were used as the $\mathrm{Zn}$ and $\mathrm{Mg}$ sources, respectively. We used $\mathrm{N}_{2} \mathrm{O}$ as the oxygen source and $\mathrm{N}_{2}$ as the carrier gas. A $0.4 \mu \mathrm{m}$-thick $\mathrm{ZnO}$ buffer layer was deposited at $450^{\circ} \mathrm{C}$, followed by a $3.3 \mu \mathrm{m}$ undoped $\mathrm{ZnO}$ layer and an undoped $\mathrm{ZnMgO}$ layer, both grown at $920^{\circ} \mathrm{C}$. The $\mathrm{ZnO}$ template exhibited a $\mathrm{Zn}$-face polarity with excellent crystal quality and layer-by-layer surface morphology. The values of full-width at half maxima (FWHM) of the $\omega$-scan rocking curves from both symmetric (0002) and asymmetric $(10 \overline{1} 1)$ planes are 186 and 622 arcsec, respectively. It clearly indicates the state-of-theart quality of the $\mathrm{ZnO}$ films with low dislocation density despite of large lattice mismatch (18\%) between $\mathrm{ZnO}$ and sapphire. The $\mathrm{Mg}$ content $(x)$ in $\mathrm{ZnMgO}$ layer was determined from the reflectance measurement of the bandgap energy of $Z_{n_{1-x}} \mathrm{Mg}_{x} \mathrm{O}$ and using the equation $E_{g}(x)=E_{g}(0)+2.145 x^{26}$.

Sample characterization. The surface morphology was characterized by the atomic force microscopy (Nanoscope IIIa, Digital Instruments Inc.) working in the tapping mode and high resolution $\mathrm{x}$-ray diffraction (HRXRD) was performed on a Panalytical
MRD diffractometer equipped with a position sensitive detector placed on the $2 \theta$ arm The $\mathrm{Cu} K \alpha 1$ radiation was selected by a flat $\mathrm{Ge}(444)$ monochromator. Capacitancevoltage $(\mathrm{C}-\mathrm{V})$ measurement was performed using mercury contacts at frequency of $1 \mathrm{MHz}$. Nanoscale analysis was carried out with a monochromated FEI Titan scanning transmission electron microscope (STEM) operated at $300 \mathrm{kV}$. Energydispersive X-ray (EDX) analysis was performed using an electron probe with a relatively large diameter of $0.5 \mathrm{~nm}$ to increase the spectral counts. Electron energyloss spectroscopy (EELS) was further utilized to probe the band structure of the heterostructure using a monochrome STEM probe with a diameter of about $1 \mathrm{~nm}$. The energy resolution of the measurement was better than $0.13 \mathrm{eV}$ and the corrections of bandgap, composition and profiling depth are the same as discussed earlier ${ }^{41}$. Temperature-dependent Hall measurements were carried out at low magnetic field of $\sim 1 \mathrm{~T}$ and temperature ranging from 10 to $300 \mathrm{~K}$. Quantum transport properties were measured in a rotator-equipped Oxford refrigerator at $1.4 \mathrm{~K}$ with the magnetic field up to $10 \mathrm{~T}$. The field-dependent Hall effect was performed at $10 \mathrm{~K}$ for sample A with B field up to $1.4 \mathrm{~T}$. by using the Lakeshore commercial Hall effect system 7707A. Both Hall effect and magnetotransport measurements are performed in a van der Pauw geometry using indium as the ohmic contact metal. The magnetic properties of sample A and $\mathrm{ZnO}$ template were measured using superconducting quantum interference devices magnetometry (SQUID) at 5 K. XPS measurements were performed using a Kratos Ultra DLD spectrometer and monochromated $\mathrm{Al} \mathrm{K} \alpha$ radiation. The atomic concentrations of each element were determined from the areas of the $\mathrm{Zn} 2 \mathrm{p}, \mathrm{Mg} 1 \mathrm{~s}, \mathrm{O} 1 \mathrm{~s}$, and $\mathrm{C} 1 \mathrm{~s}$ corelevel peaks using sensitivity factors based on spectrometer-modified Scofield crosssections.

1. Hwang, H. Y., Iwasa, Y., Kawasaki, M., Keimer, B., Nagaosa, N. \& Tokura, Y. Emergent phenomena at oxide interfaces. Nat. Mater. 11, 103-113 (2012).

2. Ohtomo, A. \& Hwang, H. Y. A high-mobility electron gas at the $\mathrm{LaAlO}_{3} / \mathrm{SrTiO}_{3}$ heterointerface. Nature 427, 423-426 (2004).

3. Thiel, S., Hammer, I. G., Schmeh, I. A., Schneider, C. W. \& Mannhart, J. Tunable quasi-two-dimensional electron gases in oxide heterostructures. Science 313, 1942-1945 (2006).

4. Tsukazaki, A. et al. Quantum hall effect in polar oxide heterostructures. Science 315, $1388-1391$ (2007)

5. Tsukazaki, A. et al. Observation of the fractional quantum Hall effect in an oxide. Nat. Mater. 9, 889-893 (2010).

6. Kozuka, Y. et al. Single-valley quantum Hall ferromagnet in a dilute $\mathrm{Mg}_{\mathrm{x}} \mathrm{Zn}_{1-\mathrm{x}} \mathrm{O} /$ $\mathrm{ZnO}$ strongly correlated two-dimensional electron system. Phys. Rev. B 85, 075302-1-075302-5 (2012).

7. Jena, D., Heikman, S., Green, D., Buttari, D. \& Coffie, R. Realization of wide electron slabs by polarization bulk doping in graded III-V nitride semiconductor alloys. Appl. Phys. Lett. 81, 4395-4397 (2002).

8. Simon, J., Protasenko, V., Lian, C. X., Xing, H. L. \& Jena, D. Polarization induced hole doping in wide bandgap uniaxial Semiconductor heterostructures. Science 327, 60-64 (2010).

9. Sundaram, M., Allen, S. J. \& Gossard, A. C. Low temperature carrier distributions in wide quantum wells of different shapes from capacitance-voltage measurements. J. Appl. Phys. 76, 1003-1007 (1994).

10. Yong, D. P. et al. High-temperature weak ferromagnetism in a low-density freeelectron. gas. Nature 397, 412-414 (1999).

11. Datta, S. \& Das, B. Electronic analog of the electro-optic modulator. Appl. Phys. Lett 56, 665-667 (1990).

12. Hall, C. R. et al. Using graded barriers to control the optical properties of $\mathrm{ZnO} /$ $\mathrm{Zn}_{0.7} \mathrm{Mg}_{0.3} \mathrm{O}$ quantum wells with an intrinsic internal electric field. Appl. Phys. Lett. 96, 193117-1-193117-3 (2010).

13. Yoon, J. G., Cho, S. W., Lee, E. \& Chung, J. S. Characteristics of indium-tin-oxide Schottky contacts to $\mathrm{ZnMgO} / \mathrm{ZnO}$ heterojunctions with band gap grading. Appl. Phys. Lett. 95, 222102-1-222102-3 (2009).

14. Rajan, S., DenBaars, S. P., Mishra, U. K., Xing, H. L. \& Jena, D. Electron mobility in graded AlGaN alloys. Appl. Phys. Lett. 88, 042103-1-042103-3 (2006).

15. Ye, J. D. et al. Two-dimensional electron gas in $\mathrm{Zn}$-polar $\mathrm{ZnMgO} / \mathrm{ZnO}$ heterostructure grown by metal-organic vapor phase epitaxy. Appl. Phys. Lett. 97, 111908-1-111908-3 (2010).

16. Pereira, S. et al. Compositional pulling effects in $\operatorname{In}_{\mathrm{x}} \mathrm{Ga}_{1-\mathrm{x}} \mathrm{N} / \mathrm{GaN}$ layers, $\mathrm{A}$ combined depth-resolved cathodoluminescence and Rutherford backscattering channelling study. Phys. Rev. B 64, 205311-1-205311-5 (2001)

17. Nishimoto, Y. et al. Plasma-assisted molecular beam epitaxy of high optical quality $\mathrm{MgZnO}$ films on $\mathrm{Zn}$-polar $\mathrm{ZnO}$ substrates. Appl. Phys. Express 1, 091202 1-091202-3 (2008).

18. Matsui, H., Tabata, H., Hasuike, N. \& Harima, H. Critical thickness and lattice relaxation of Mg-rich strained $\mathrm{Mg}_{0.37} \mathrm{Zn}_{0.63} \mathrm{O}$ (0001) layers towards multiquantum-wells. J. Appl. Phys. 99, 024902-1-024902-7 (2006).

19. Sawai, Y., Hazu, K. \& Chivhibu, S. F. Surface stoichiometry and activity control for atomically smooth low dislocation density $\mathrm{ZnO}$ and pseudomorphic $\mathrm{MgZnO}$ epitaxy on a $\mathrm{Zn}$-polar $\mathrm{ZnO}$ substrate by the helicon-wave-excited-plasma sputtering epitaxy method. J. Appl. Phys. 108, 063541-1-063541-8 (2010).

20. Allen, M. W. et al. Polarity effects in the $\mathrm{x}$-ray photoemission of $\mathrm{ZnO}$ and other wurtzite semiconductors. Appl. Phys. Lett. 98, 101906-1-101906-3 (2011). 
21. Dulub, O., Diebold, U. \& Kresse, G. Novel stabilization mechanism on polar surface: $\mathrm{ZnO}(0001)-\mathrm{Zn}$. Phys. Rev. Lett. 90, 016102-1-016102-4 (2003).

22. Torbrugge, S., Ostendorf, F. \& Reichling, M. Stabilization of Zinc-terminated ZnO (0001) by a modified surface stoichiometry. J. Phys. Chem. C 113, 4909-4914 (2009).

23. Lai, J. H. et al. Stabilization of $\mathrm{ZnO}$ polar plane with charged surface nanodefects. Phys. Rev. B 82, 155406-1-155406-5 (2010).

24. Snider, G. L. 1D Poisson, URL, http//www.nd.edu/ gsnider/

25. Furno, E. et al. Electronic and optical properties of $\mathrm{Mg}_{\mathrm{x}} \mathrm{Zn}_{1-\mathrm{x}} \mathrm{O}$ and $\mathrm{Be}_{\mathrm{x}} \mathrm{Zn}_{1-\mathrm{x}} \mathrm{O}$ quantum wells. J. Electron. Mater. 39, 936-942 (2010)

26. Tampo, H. et al. Polarization-induced two-dimensional electron gases in $\mathrm{ZnMgO} /$ ZnO heterostructures. Appl. Phys. Lett. 93, 202104-1-202104-3 (2008).

27. Wang, X. F. et al. Terahertz time-domain magnetospectroscopy of a high-mobility two-dimensional electron gas. Opt. Lett 32, 1845-1847 (2007).

28. Jena, D., Gossard, A. C. \& Mishra, U. K. Dipole scattering in polarization induced III-V nitride two-dimensional electron gases. J. Appl. Phys. 88, 4734-4738 (2000).

29. Murzin, S. S., Jansen, A. G. M. \& Linden, P. Quantization of the Hall Conductance in a three-dimensional layer. Phys. Rev. Lett. 80, 2681-2684 (1998).

30. Zeng, Y. J. et al. Tuning quantum corrections and magnetoresistance in $\mathrm{ZnO}$ nanowires by ion implantation. Nano Lett. 12, 666-672 (2012).

31. Sergio, C. S. et al. Coexistence of a two- and three-dimensional Landau states in a wide parabolic quantum well. Phys. Rev. B 64, 115314-1-115314-9 (2001).

32. Smith, J. L. \& Stiles, P. J. Electron-Electron Interactions Continuously Variable in the Range 2.1 $>\mathrm{r}_{\mathrm{s}}>0$.9. Phys. Rev. Lett. 29, 102-104 (1972).

33. Yan Voon, L. C., Willatzen, M., Cardona, M. \& Christensen, N. E. Terms linear in $\mathrm{k}$ in the band structure of wurtzite-type semiconductors. Phys. Rev. B 53, 1070310714 (1996)

34. Shalom, M., Tai, C. W., Lereah, Y., Sachs, M. \& Levy, E. Anisotropic magnetotransport at the $\mathrm{SrTiO}_{3} / \mathrm{LaAlO}_{3}$ interface. Phys. Rev. B 80, 140403-1140403-4 (2009)

35. Brinkman, A., Huigben, M., Zalk, M. V., Huijben, J. \& Zeitler, U. Magnetic effects at the interface between non-magnetic oxides. Nat. Mater. 6, 493-496 (2007).

36. Zong, F. H., Lin, C. \& Ceperley, D. M. Spin polarization of the low-density threedimensional electron gas. Phys. Rev. E 66, 036703-1-036703-7 (2002).

37. Bert, J. A. et al. Direct imaging of the coexistence of ferromagnetism and superconductivity at the $\mathrm{LaAlO}_{3} / \mathrm{SrTiO}_{3}$ interface. Nat. Phys. 7, 767-771 (2011).

38. Wang, Q., Sun, Q., Chen, G., Kawazoe, Y. \& Jena, P. Vacancy-induced magnetism in ZnO thin film and nanowires. Phys. Rev. B 77, 205411-1-205411-7 (2008).

39. Fischer, G. et al. Room-temperature p-induced surface ferromagnetism: Firstprinciles study. Phys. Rev. B 84, 205306-1-205306-5 (2011).
40. Yoshimatsu, K., Yasuhara, R., Kumigashira, H. \& Oshima, M. Origin of metallic states at the heterointerface between the band insulators $\mathrm{LaAlO}_{3}$ and $\mathrm{SrTiO}_{3}$. Phys. Rev. Lett 101, 026802-1-026802-4 (2008).

41. Bosman, M. et al. Nanoscale band gap spectroscopy on $\mathrm{ZnO}$ and GaN-based compounds with a monochromated electron microscope. Appl. Phys. Lett. 95, 101110-1-101110-3 (2009)

\section{Acknowledgements}

The authors gratefully acknowledge Dr. James Lloyd-Hughes and Dr. Samuel Jones from Oxford University for their measurements and helpful discussion of THz-TDS results and Dr. Ning Tang from Peking University for his valuable suggestions on the SdH oscillations. This work is supported by the Singapore Agency for Science, Technology, and Research (A*STAR), under SERC Grant No. 0521260095, the Australian Research Council Discovery Project Grant (DP1096918) and the State Key Program for Basic Research of China under Grant No. 2011 CB302003 and Basic Research Program of Jiangsu Province (BK2011437)

\section{Author contributions}

M. B. carried out the TEM and EELS experiments; S. L. G. and Y. D. Z. performed Hall measurement; S. T. L. carried out the magnetotransport, XPS and SQUID experiments; J. D. Y. and K. L. T. designed the research, carried out all the experiments, analysed the data and co-wrote the manuscript, which H. H. T., C. J. and X.W. S. edited. All authors discussed the results and commented on the manuscript.

\section{Additional information}

Supplementary information accompanies this paper at http://www.nature.com/ scientificreports

Competing financial interests: The authors declare no competing financial interests.

License: This work is licensed under a Creative Commons

Attribution-NonCommercial-ShareAlike 3.0 Unported License. To view a copy of this license, visit http://creativecommons.org/licenses/by-nc-sa/3.0/

How to cite this article: Ye, J. et al. Spin-polarized Wide Electron Slabs in Functionally Graded Polar Oxide Heterostructures. Sci. Rep. 2, 533; DOI:10.1038/srep00533 (2012). 